Manuscript submitted to Biochemistry

DOI of published article: https://doi.org/10.1021/acs.biochem.7b00477

\title{
Protein dynamics in the reductive activation of a B12-containing enzyme.
}

Sebastian Goetzl ${ }^{\dagger}$, Christian Teutloff $^{\ddagger}$, Tobias Werther $^{\dagger}$, Sandra E. Hennig ${ }^{\dagger}$, Jae-Hun Jeoung ${ }^{\dagger}$, Robert Bittl ${ }^{\ddagger}$ and Holger Dobbek ${ }^{\dagger} *$

${ }^{\dagger}$ Institut für Biologie, Strukturbiologie/Biochemie, Humboldt-Universität zu Berlin, Berlin, Germany

‡ Fachbereich Physik, Freie Universität Berlin, Berlin, Germany

To whom correspondence should be addressed

*holger.dobbek@hu-berlin.de

Funding Source Statement:

HD acknowledges the DFG for funding of the project through grant DO 785/5-1 and 5-2. All authors acknowledge funding by the cluster of excellence "Unifying Concepts in Catalysis UniCat" (EXC 314). 


\section{ABBREVIATIONS}

CfsA, large subunit of CoFeSP;

CfsB, small subunit of CoFeSP;

CoFeSP, corrinoid iron/sulfur protein;

DEER, double electron-electron resonance;

$E P R$, electron paramagnetic resonance;

$\mathrm{ET}$, electron transfer;

FRET, Förster resonance energy transfer;

ITC, isothermal titration calorimetry;

MetH, methionine synthase;

MTSL,

(S-(1-oxyl-2,2,5,5-tetramethyl-2,5-dihydro-1H-pyrrol-3-yl)methyl

methanesulfonothioate,

PELDOR, pulsed electron-electron double resonance;

RACo, reductive activator of CoFeSP. 


\section{ABSTRACT}

B12-dependent proteins are involved in methyl transfer reactions ranging from the biosynthesis of methionine in humans to the formation of acetyl-CoA in anaerobic bacteria. During their catalytic cycle they undergo large conformational changes to interact with various proteins. Recently, the crystal structure of the B12-containing corrinoid iron sulfur protein (CoFeSP) in complex with its reductive activator (RACo) was determined, providing a first glimpse on how energy is transduced in the ATP-dependent reductive activation of corrinoid-containing methyltransferases. The thermodynamically uphill electron transfer from RACo to CoFeSP is accompanied by large movements of the cofactor-binding domains of CoFeSP. To refine the structure-based mechanism we analyzed the conformational change of the B12-binding domain of CoFeSP by pulsed electron-electron double resonance (PELDOR) and Förster resonance energy transfer (FRET) spectroscopy. We show that the site-specific labels on the flexible B12-binding domain and the small subunit of CoFeSP approach by $11 \AA$ in the RACo:CoFeSP complex, consistent with the recent crystal structures. By analyzing the transient kinetics of formation and dissociation of the RACo:CoFeSP complex, we determined rate constants of $0.75 \mu \mathrm{M}^{-1} \mathrm{~s}^{-1}$ and $0.33 \mathrm{~s}^{-1}$ for $k_{\text {on }}$ and $k_{\text {off, }}$ respectively. Our results indicate that the large movement observed in crystals also occur in solution and that neither the formation of the protein encounter complex nor the large movement of the B12-binding domain are rate-limiting for the ATP-dependent reductive activation of CoFeSP by RACo. 


\section{INTRODUCTION}

Metalloenzymes depend for their activity on a defined oxidation state and inactive redox states have to be reactivated. B12-dependent proteins such as the corrinoid iron-sulfur containing protein (CoFeSP) and methionine synthase (MetH) facilitate the transfer of a methyl group for biosynthesis of acetyl-CoA and methionine, respectively. The tightly bound cobalamin cofactor acts as methyl group acceptor and donor by cycling between the methylated $\mathrm{CH}_{3}-\mathrm{Co}(\mathrm{III})$ and the reduced $\mathrm{Co}(\mathrm{I})$ state, the latter state is susceptible to oxidation to the inactive $\mathrm{Co}(\mathrm{II})$ state. $^{1}$

We recently described an ATP dependent reductive activator of CoFeSP (RACo), ${ }^{2}$ which is a member of a diverse family of reductive activators of B12-containing methyltransferases..$^{3-6}$ RACo reduces the corrinoid cofactor of CoFeSP, thereby regenerating the active Co(I) state. To achieve the thermodynamic uphill electron transfer from a reduced [2Fe2S] cluster in RACo to the Co(II) ion in CoFeSP, RACo couples electron transfer to the hydrolysis of ATP (eq. 1). ${ }^{2}$

$\mathrm{Co}(\mathrm{II}) \mathrm{FeSP}+\left\{\mathrm{RACo}[2 \mathrm{Fe} 2 \mathrm{~S}]^{1+}\right\}+\mathrm{MgATP} \Leftrightarrow \mathrm{Co}(\mathrm{I}) \mathrm{FeSP}+\left\{\mathrm{RACo}[2 \mathrm{Fe} 2 \mathrm{~S}]^{2+}\right\}+\mathrm{MgADP}+\mathrm{Pi}(1)$

RACo and inactive CoFeSP form a stable complex, whose dissociation constant was estimated using isothermal titration calorimetry (ITC) $\left(K_{d}=18 \mathrm{nM}\right) .{ }^{7,8}$ Complex formation depends on the oxidation state of $\mathrm{Co}$, alters the electronic structure of the corrinoid cofactor and enhances the ATPase activity of RACo. ${ }^{7-9}$ These changes are enabled by large structural rearrangements in RACo and both cofactor-binding domains of CoFeSP. ${ }^{8}$ The B12-binding domain is rotated by $\sim 45^{\circ}$, displacing the Co-ion by $20 \AA$ in crystals of the RACo:CoFeSP complex (Figure 1A). The [4Fe4S]-cluster domain of CoFeSP is shifted by $42 \AA$ and binds near the dimerization interface of RACo (Figure 1). Both movements together decrease the distance between Co and the [4Fe4S]-cluster from $60 \AA$ to $30 \AA .^{8}$ However, it is unknown if these large movements were only favored by crystal packing interactions or also occur in solution. 


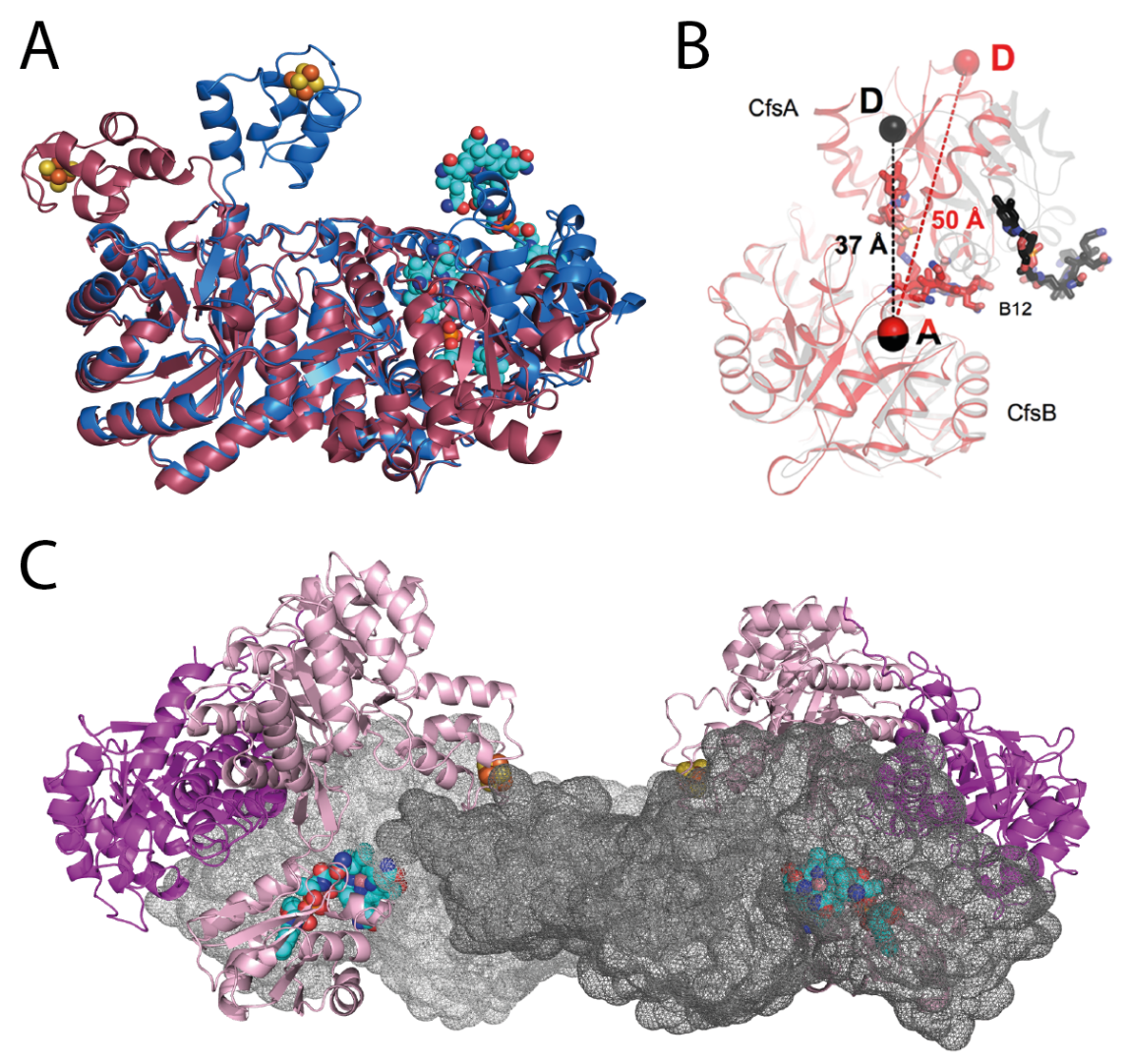

Figure 1. Conformational changes of CoFeSP upon forming a complex with RACo. (A) Complex formation induces large conformational changes in the two cofactor-containing domains of the CfsA subunit of CoFeSP. Displayed are the conformations of CoFeSP alone (dark red) and CoFeSP in complex with RACo (blue). (B) Position of labels. Superimposition of CoFeSP in the free state (PDB 2YCL, ${ }^{10,11}$ colored in grey) with CoFeSP from the RACo:CoFeSP complex structure (PDB 4C1N, ${ }^{8}$ colored in red). The C $\alpha$-atoms of cysteine residues used to attach the donor (D) and acceptor dyes (A) are highlighted as spheres. The distance between the C $\alpha$ atoms of E138 (CfsB subunit) and C $\alpha$ of E397 (CfsA subunit) is $50 \AA$ in CoFeSP and $37 \AA$ in the complex, respectively. Donor and acceptor positions are remote from the complex interface of the RACo:CoFeSP complex. (C) Overall structure of the RACo:CoFeSP complex (PDB 4C1N). ${ }^{8}$ The RACo dimer is indicated by light and dark grey mesh surfaces. The two subunits of CoFeSP are colored light pink ( $\mathrm{Cfs} A$ ) and dark pink ( $\mathrm{CfsB}$ ). The [2Fe2S]-cluster of RACo has not been resolved in the crystal structure. ${ }^{8}$

The purpose of this paper is to investigate the conformational changes in solution and to compare them to the movements observed in crystals. By combining distance resolving PELDOR measurements with time-resolved measurements using stopped-flow FRET spectroscopy, we confirm the extent of the conformational changes observed in crystals and show that they are kinetically competent and not limiting the rate of reductive activation. 


\section{EXPERIMENTAL PROCEDURES}

\section{$\underline{\text { Materials }}$}

All chemicals were purchased from Sigma (Taufkirchen, Germany), AppliChem (Darmstadt, Germany) and Roth (Karlsruhe, Germany) and were at least of analytical grade. $\mathrm{N}_{2}$ and $\mathrm{N}_{2} / \mathrm{H}_{2}$ (95\%/5\%) gases were obtained from Air Liquide (Berlin, Germany). Enzymes (DNA polymerases, DNA ligases and restriction enzymes) used in molecular biology experiments were purchased from either Fermentas (St. Leon-Roth, Germany) or Finnzyme (Thermo Fisher, Schwerte, Germany). The fluorescent labels ATTO 488 and ATTO 590 were acquired from ATTO-TEC (Siegen, Germany) as cysteine-reactive maleimides. The (S-(1-oxyl-2,2,5,5tetramethyl-2,5-dihydro-1H-pyrrol-3-yl)methyl methanesulfonothioate (MTSL) spinlabel was purchased from Biomol GmbH (Hamburg, Germany). All buffer solutions were made anoxic in bottles with butyl rubber septum by at least 4 cycles of evacuating and flushing with $\mathrm{N}_{2}$ gas at a vacuum-gas line. Protein purification, the preparation of bilabeled CoFeSP and UV/vis spectroscopy were performed under anoxic conditions under an atmosphere of $95 \%$ $\mathrm{N}_{2} / 5 \% \mathrm{H}_{2}$ (glove box model B, COY Laboratory Products Inc., Michigan, USA). Stopped-flow and ITC experiments were conducted under a pure $\mathrm{N}_{2}$ atmosphere (MBraun LABstar glovebox) with oxygen levels below $0.5 \mathrm{ppm}$.

\section{Molecular biology}

The genes acs $C$ and $a c s D$, encoding the large (CfsA) and the small ( $C f s B)$ subunit of CoFeSP from Carboxydothermus hydrogenoformans were amplified by PCR as described previously. ${ }^{10} \mathrm{Ncol} / \mathrm{Notl}$ digested $\operatorname{acs} \mathrm{C}$ and $\mathrm{Ndel} / \mathrm{Xhol}$ digested $\operatorname{acs} D$ were ligated into pETDuet-1 and named pPKacsC and PPKacsD, respectively. Suitable positions for FRETlabeling were identified by visual inspection of the CoFeSP structure ( $p d b$ : $2 Y C L$ ) using COOT. ${ }^{12}$ For site-specific labeling of CoFeSP, two glutamate residues (E397 in CfsA and E138 in $\mathrm{CfsB}$ ) were replaced by cysteine residues, whereas a surface exposed cysteine residue (C348) in CfsA was replaced by serine. The PPKacsC-C348S/E397C plasmid, encoding the CfsA-C348S/E397C variant and pPKacsD-E138C, encoding the CfsB-E138C variant were prepared by following the QuickChange site-directed mutagenesis protocol from Stratagene and analyzed by DNA sequencing (Eurofins MWG Operon, Germany). The following specific primer pairs were used for mutagenesis: 
CfsA:

C348S: $\quad$ 5'-CTC GTT GAC TTA CTT CTC TGT TGC CGG TGA TGT AG-3'

5'-CCT TCT ACA TCA CCG GCA ACA GAG AAG TAA GTC AAC GAG AAG-3'

E397C: $\quad$ 5'-GGA AAG CGG AAT TGC TIG CAA AGT TAA CCA CAG AAA AGC-3'

5'-GCT TTT CTG TGG TTA ACT TTG CAA GCA ATT CCG CTT TCC-3'

CfsB:

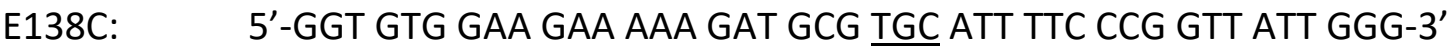

5'-CCC AAT AAC CGG GAA AAT GCA CGC ATC TTT TTC TTC CAC ACC-3'

The recombinant plasmids pPKacsC-C348S/E397C and pPKacsD-E138C were transformed into $E$. coli $\mathrm{BL} 21(\mathrm{DE} 3)$. Both cultures were grown under stirring at $37^{\circ} \mathrm{C}$ in $\mathrm{mTB}$ media ${ }^{13}$ containing carbenicilin $(50 \mu \mathrm{g} / \mathrm{ml})$. When $\mathrm{OD}_{600}$ of the culture reached 0.7 , gene expression was induced by adding isopropyl $\beta$-D-thiogalactopyranoside (final concentration $0.2 \mathrm{mM}$ ). Cultures expressing acsC-C348S/E397C were supplemented with $0.25 \mathrm{mM} \mathrm{FeSO}_{4}$ and $\mathrm{Na}_{2} \mathrm{~S}$ after induction. The cultures were further incubated for $5 \mathrm{~h}$ at $37^{\circ} \mathrm{C}$ and harvested by centrifugation at $8,000 \mathrm{rpm}$ and $4{ }^{\circ} \mathrm{C}$ for $20 \mathrm{~min}$. Cell pellets were stored at $-20^{\circ} \mathrm{C}$ until use. For preparation of the reductive activator of CoFeSP (RACo) the recombinant plasmid pPKCoDuet1TEV was transformed into E. coli BL21(DE3) and expressed as described previously. $^{2}$

\section{Purification of CfsB-E138C}

$10 \mathrm{~g}$ of frozen cells were resuspended in buffer $\mathrm{A}(50 \mathrm{mM}$ Tris- $\mathrm{HCl}, \mathrm{pH} 8.0$ ) and disrupted by sonication in an ice-cooled glass rosette. After ultracentrifugation for $1 \mathrm{~h}$ at 35,000 rpm and $8{ }^{\circ} \mathrm{C}$, the clear supernatant was loaded on a DEAE column $(50 \mathrm{~mL})$, equilibrated with buffer A. After washing with buffer A, protein was eluted with $500 \mathrm{~mL}$ of a linear gradient from 0 $0.6 \mathrm{M} \mathrm{NaCl}$ in buffer A. Colorless fractions containing CfsB-E138C were combined, supplemented with $1 \mathrm{M}$ ammonium sulfate and incubated for $12 \mathrm{~h}$ at $4{ }^{\circ} \mathrm{C}$. Precipitated protein was removed by centrifugation. The clear supernatant was applied to a phenylsepharose column $(20 \mathrm{~mL})$ equilibrated with buffer $A$ containing $1 \mathrm{M}$ ammonium sulfate. After washing, proteins were eluted with $200 \mathrm{~mL}$ of a linear gradient of $1-0 \mathrm{M}$ ammonium sulfate in buffer A. Fractions containing CfsB-E138C were combined and concentrated to a volume of $2 \mathrm{~mL}$ using a Vivaspin 70 (10 kDa MWCO; Vivascience GmbH) spin concentrator. Concentrated CfsB-E138C was further purified by size exclusion chromatography, using a 
$120 \mathrm{~mL}$ Superdex ${ }^{\mathrm{TM}} 200$ prep-grade gel filtration column (GE Health Care) equilibrated in

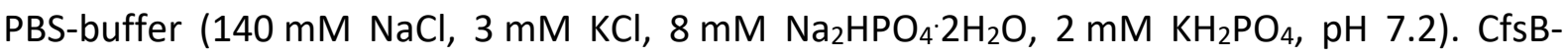
E138C solution was reduced with $3 \mathrm{mM}$ DT, concentrated and frozen in liquid nitrogen.

\section{Purification of CfSA-C348S/E397C}

$13 \mathrm{~g}$ of frozen cells were dissolved in $70 \mathrm{~mL}$ of PBS-buffer containing $2 \mathrm{mM}$ DTT. After $15 \mathrm{~min}$ sonication, the cell lysate was centrifuged for $1.5 \mathrm{~h}$ at $35,000 \mathrm{rpm}$ and $10^{\circ} \mathrm{C}$. The colored, CfsA free supernatant was discarded, while the pellet containing insoluble protein was successively washed with PBS-buffer containing $1 \mathrm{mM}$ EDTA and centrifuged until the supernatant became colorless. Finally, the pellet was resuspended in $10 \mathrm{~mL}$ buffer $A$ containing $1 \mathrm{mM}$ DTT and frozen at $-80^{\circ} \mathrm{C}$.

\section{Preparation of site-specific, bilabeled CoFeSP}

All steps including work with fluorescent dyes were performed under dim light. $150 \mu \mathrm{M}$ of CfsB-E138C in $1 \mathrm{~mL}$ PBS-buffer were incubated for $2 \mathrm{~h}$ at $25^{\circ} \mathrm{C}$ with 1.5 molar excess of ATTO 590 fluorescent dye, dissolved in water-free DMSO. Excess or unreactive dye was removed by gel filtration ( $120 \mathrm{ml}$ Superdex ${ }^{\mathrm{TM}} 200$ prep-grade, GE Health Care). 2 mg of labeled CfsBE138C in $0.45 \mathrm{~mL}$ PBS-buffer were mixed with $0.45 \mathrm{~mL}$ CfSA-C348S/E397C pellet suspension and supplemented with $0.1 \mathrm{~mL}$ of a $2 \mathrm{mM}$ hydroxocobalamin solution. After addition of $1 \mathrm{~mL}$ PBS-buffer containing $4 \mathrm{M}$ urea, the protein mix was incubated for $15 \mathrm{~min}$ at $25^{\circ} \mathrm{C}$. After centrifugation for 10 minutes at $12,000 \mathrm{rpm}$, the clear supernatant was diluted and concentrated to a final concentration of $0.1 \mathrm{M}$ urea. To complete corrinoid reconstitution, $200 \mu \mathrm{M}$ hydroxocobalamin was added. After $1 \mathrm{~h}$, the mix was applied to a gel filtration column (120 mL Superdex ${ }^{T M} 200$ prep-grade, GE Health Care). The peak fractions corresponding to a complex between ATTO 590 labeled CfsB-E138C and CfsA-C348S/E397C were combined and concentrated. A 1.5 molar excess of ATTO 488 dye was added and after $2 \mathrm{~h}$ incubation at $25{ }^{\circ} \mathrm{C}$ another gel filtration was performed as described above. The sitespecific bilabeled CoFeSP variant (CoFeSP ${ }^{\ddagger}$ ) was concentrated, frozen and stored in liquid nitrogen until use.

\section{Preparation of RACo}


Frozen cells resuspended in buffer A were broken by sonication. The cell suspension was cleared from cell debris by ultracentrifugation at $35,000 \mathrm{rpm}$ and $12{ }^{\circ} \mathrm{C}$ for $1 \mathrm{~h}$. The supernatant was loaded on a Ni-sepharose high-performance column $(5 \mathrm{~mL})$ equilibrated with buffer A containing $25 \mathrm{mM}$ imidazole and $200 \mathrm{mM} \mathrm{NaCl}$. The protein was eluted with a $50 \mathrm{~mL}$ gradient of 25-250 mM imidazole in buffer A. Colored fractions were pooled, diluted four fold and loaded on a Q-sepharose column $(50 \mathrm{~mL})$ equilibrated with buffer $A$. After washing of the column, a linear $450 \mathrm{~mL}$ gradient of $0-1 \mathrm{M} \mathrm{NaCl}$ was applied to elute the protein. The purification tag was cleaved by catalytic amounts of TEV protease and RACo was further purified by size exclusion chromatography as described earlier. ${ }^{4}$

\section{Rapid reaction techniques}

Transient kinetics were measured under anoxic conditions using a BioLogic SFM400 stoppedflow system with a $1.5 \mathrm{~mm}$ path length FC-15 cuvette coupled to a monochromator and photomultiplier. Kinetics were measured under strictly anoxic conditions $\left(<0.5 \mathrm{ppm} \mathrm{O}_{2}\right)$ in an anoxic glove box (MBraun, Garching, Germany) housing the stopped-flow system. The reaction temperature of $25^{\circ} \mathrm{C}$ was controlled with a Haake SC100-A10 thermostat thermally equilibrated with a home-made water circulation system inside the glove box. For all experiments, the FRET donor was excited at $450 \mathrm{~nm}$ and the acceptor fluorescence was monitored for an appropriate time scale using a $590 \mathrm{~nm}$ long-pass filter (Andover Corporation). The formation of the CoFeSP:RACo complex was analyzed by mixing constant concentrations of CoFeSP $P^{\ddagger}$ in buffer A containing $100 \mathrm{mM} \mathrm{NaCl}$ with various concentrations of RACo, yielding final concentrations of 0.25 and $0.75 \mu \mathrm{M}$ CoFeSP ${ }^{\ddagger}$ and 2.5 to $30 \mu \mathrm{M}$ RACo, respectively. Each experiment was repeated at least three times for each RACo concentration. The observed kinetic transients were fitted to eq 2 using BioKine (BioLogic).

$$
\mathrm{Fl}(\mathrm{t})=\mathrm{a} * \mathrm{t}+\mathrm{b}+\mathrm{c}_{1} * \mathrm{e}^{-k o b s 1 * \mathrm{t}}+\mathrm{c}_{2} * \mathrm{e}^{-k o b s 2 * \mathrm{t}}
$$

where $\mathrm{Fl}(\mathrm{t})$ is the time-dependent fluorescence, $\mathrm{c}_{1}$ and $\mathrm{c}_{2}$ are the amplitudes and $k_{\mathrm{obs}}$ and $k_{\mathrm{obs} 2}$ are two observed rate constants. Data were interpreted assuming a single-step binding mechanism (eq 3).

$$
\mathrm{A}+\mathrm{B} \underset{k_{\text {off }}}{\stackrel{k_{\text {on }}}{\rightleftharpoons}} \mathrm{C}
$$

where $\mathrm{A}$ is CoFeSP${ }^{\ddagger}, \mathrm{B}$ is RACo, C is the CoFeSP ${ }^{\ddagger}$ :RACo complex and $k_{\text {on }}$ and $k_{\text {off }}$ are the association and dissociation rate constants, respectively. RACo concentrations were at least 
tenfold higher than concentrations of CoFeSP ${ }^{\ddagger}$, resulting in pseudo-first conditions. Binding rate constants were derived from the association assay using linear equation eq $4^{14}$

$$
k_{\mathrm{obs}}=k_{\mathrm{off}}+k_{\mathrm{on}} *[\mathrm{~B}]
$$

where $[B]$ is the concentration of RACo.

The transient kinetics of complex dissociation was measured in a competitive displacement experiment. An equimolar mixture of $1 \mu \mathrm{M}$ CoFeSP ${ }^{\ddagger}$ and RACo in buffer $\mathrm{A}$ containing $100 \mathrm{mM} \mathrm{NaCl}$ was rapidly mixed with a 50 -fold molar excess of unlabeled CoFeSP and the fluorescence signal was monitored as described above. The signal change was approximated using a single-exponential function. Under these conditions, the observed rate constants approximates the dissociation rate constant of the labelled species (CoFeSP ${ }^{\ddagger}$ ) dissociating from the complex. ${ }^{15}$

\section{Size exclusion chromatography}

To investigate complex formation between RACo and the CoFeSP variant (CoFeSP*), an analytical gelfiltration experiment was performed as described previously. ${ }^{2}$ Equimolar concentrations of RACo and CoFeSP* $(20 \mu \mathrm{M})$ in $500 \mu \mathrm{l} 20 \mathrm{mM}$ Tris- $\mathrm{HCl}$ pH 8.0 were incubated for 20 minutes and loaded on a Superdex ${ }^{\mathrm{TM}} 200$ prep-grade gel filtration column.

\section{$\underline{\text { Reductive activation assay }}$}

The ATP-dependent electron transfer from RACo to Co(II)-CoFeSP was measured as described previously. ${ }^{2} 2.5 \mu \mathrm{M}$ RACo were reduced by $5 \mathrm{mM}$ DTT and the electron transfer to $2.5 \mu \mathrm{M}$ wildtype CoFeSP or CoFeSP* was initiated by adding $1 \mathrm{mM} \mathrm{Mg-ATP.} \mathrm{Co(I)} \mathrm{formation} \mathrm{was}$ measured by following the increase in absorption at $390 \mathrm{~nm}$ on an Agilent 8453 photodiode array spectrophotometer.

\section{$\underline{\text { PELDOR measurements }}$}

For the EPR/PELDOR experiments, purified CoFeSP was labeled the positions E138C/E397C by incubation with a 20-fold excess of MTSL for $14 \mathrm{~h}$ at $25^{\circ} \mathrm{C}$. Excess MTSL was removed using a PD10 column.

The final protein concentrations were at least $100 \mu \mathrm{M}$. The proteins were buffer-exchanged with $20 \mathrm{mM}$ deuterated Tris- $\mathrm{HCl}$, buffer, 1:1 mixed with deuterated glycerol allowing for longer relaxation times and therefore larger observation time windows for the PELDOR 
experiments. The protein was then filled into quartz capillaries $(3.9 \mathrm{~mm}$ o.d., QSil, Ilmenau/Germany) and quickly frozen in precooled ethanol.

Pulsed EPR experiments were carried out at a Bruker Elexsys E680 spectrometer operating at a frequency of $9.7 \mathrm{GHz}$, equipped with a second frequency option (E580-400U), a $1 \mathrm{~kW}$ TWT (Applied System Engineering, USA), and a MD5 dielectric ring resonator. Temperature adjustment was achieved by using a helium cryostat (CF935) and an ITC5 temperature controller (both Oxford Instruments, UK). All experiments were carried out at $40 \mathrm{~K}$.

For the PELDOR measurements a 4-pulse sequence ${ }^{16,17}$ with $\pi$-pulses of $32 \mathrm{~ns}$ for the observer and for the pump frequency was used. Pumping of spins was realized in the center of the EPR spectrum, observation was done on the left shoulder, with a frequency offset of $70 \mathrm{MHz}$. A two-step phase cycle was applied, and a $\tau$-stepping procedure between the first two pulses ( 8 steps of $8 \mathrm{~ns}$ increments) in order to reduce nuclear echo modulations occurring as artifacts in the PELDOR spectrum.

The raw PELDOR spectra were processed by DeerAnalysis 2013.2, a toolbox for Matlab, ${ }^{17}$ employing an exponential baseline correction for the background. Distance distributions were generated by Tikhonov regularization as implemented in the toolbox, using a regularization parameter of 100 . In silico distance distributions from the known crystal structures (PDB: 2YCL for CoFeSP, and PDB: 4C1N for CoFeSP:RaCo) were generated by MMM2013.2, a Matlab Toolbox for structural modeling of macromolecular systems. ${ }^{18}$

\section{RESULTS}

To follow movements of the flexible B12-binding domain, we produced a CoFeSP variant with three amino acid exchanges, suitable for site-specific labeling with thiol-reactive moieties. E397 at the top of the B12-binding domain of the large CoFeSP subunit (CfsA) and E138 at the rigid small CoFeSP subunit (CfsB) were replaced by cysteine residues (Figure 1). C348, a surface exposed cysteine on CfsA, was changed to serine. Both subunits were individually expressed and purified and the complex reassembled. Finally, the corrinoid cofactor was inserted by reconstitution. The CoFeSP variant (CoFeSP*) showed the same behavior as native CoFeSP: it formed a complex with RACo (Figure S1) and ATP-dependent electron transfer from RACo to CoFeSP* occurred with an observed rate constant ( $\left.k_{\text {obs }}\right)$ of $0.044 \pm 0.006 \mathrm{~s}^{-1}$ (Figure S2; native CoFeSP: $k_{\mathrm{obs}}$ of $0.038 \pm 0.013 \mathrm{~s}^{-1}$ ). 
To analyze the movement of the B12-binding domain of CoFeSP, we attached MTSL spinlabels to CoFeSP* and used PELDOR spectroscopy (using the 4-pulse DEER sequence) ${ }^{16}$ to quantify the inter-spin distances for labeled CoFeSP* and the RACo:CoFeSP* complex in solution.

The distance distributions were obtained by employing the Tikhonov regularization method (Figure 2) implemented in DEERAnalysis. ${ }^{17}$ The main inter-spin distance of labeled CoFeSP* is $5.8 \mathrm{~nm}$. The main label distance decreases by $1.1 \mathrm{~nm}$ to $4.7 \mathrm{~nm}$ in the RACo:CoFeSP* complex, which is consistent with the observed movement of $1.3 \mathrm{~nm}$ in the crystal structures (Figure 1). A simulation of the distance distribution of MTSL rotamers, assuming flexible side chains, agrees well with the experimental PELDOR data (Figures 2B, S3 and S4). It is important to note that the width of the predicted distance distribution is due to the flexibility of the linker and represents a rotamer distribution for the spin-labeled side chain and that the main intensity of the experimental distance distribution falls well within the predicted distribution.
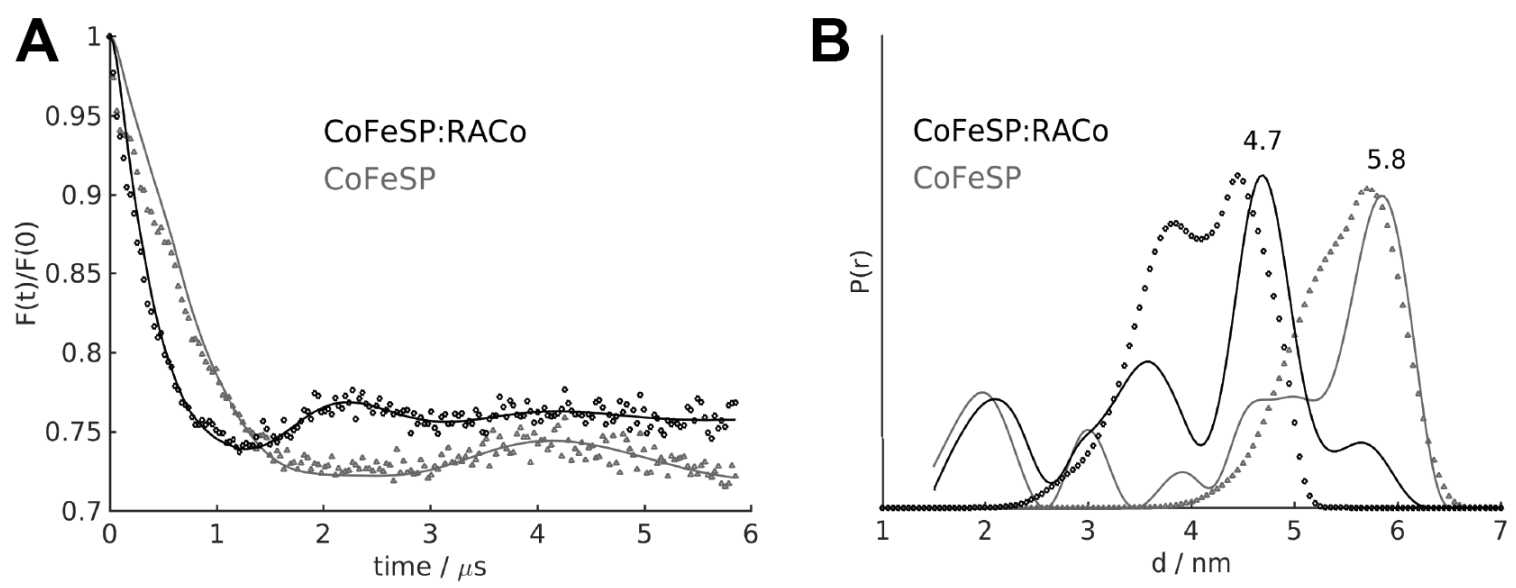

Figure 2. PELDOR data and distance distribution. (A) Phase and background corrected data for CoFeSP (grey triangles) and RACo:CoFeSP complex (black circles). The simulated time traces are shown as solid lines. (B) Inter-spin distance distributions obtained by Tikhonov regularization (solid lines) and modeled distance distributions obtained by MMM using the protein structures from 2YCL (CoFeSP, triangles) and 4C1N (RACo:CoFeSP, circles). Spectra were recorded at $40 \mathrm{~K}$.

FRET spectroscopy was employed to follow the rate of conformational changes. The fluorescent dyes Atto 488 (donor) and Atto 590 (acceptor) were coupled to the same reactive cysteines as employed for the PELDOR study by following standard procedures. The Atto 488/590 bi-labeled CoFeSP* (CoFeSP ${ }^{\ddagger}$ ) shows absorption peaks around 505 and $601 \mathrm{~nm}$, 
corresponding to the absorption maxima of Atto 488 and Atto 590, respectively (Figure S5). When CoFeSP-bound Atto 488 is excited at $450 \mathrm{~nm}$, Atto 590 emits at $620 \mathrm{~nm}$ (Figure S5, inset). The $620 \mathrm{~nm}$ peak is missing when the free dyes in solution are excited at $450 \mathrm{~nm}$. When RACo is added to bi-labeled CoFeSP, emission at $620 \mathrm{~nm}$ increases (Figure S5), indicating a higher FRET efficiency, as expected from the approach of the MTSL spin label by $1.1 \mathrm{~nm}$ and the shorter distance in the RACo:CoFeSP crystal structure compared to the structure of CoFeSP (Figure 1). Neither donor only, nor acceptor only samples showed an increasing fluorescence signal after RACo addition.

Complex formation between CoFeSP ${ }^{\ddagger}$ and RACo was followed by stopped-flow FRET spectroscopy (Figure $3 \mathrm{~A})$ under anoxic conditions $\left(\left[\mathrm{O}_{2}\right]<0.5 \mathrm{ppm}\right)$. Atto 488 was excited at $450 \mathrm{~nm}$ and the acceptor fluorescence was monitored using a $590 \mathrm{~nm}$ longpass filter. Constant concentrations of $0.25 \mu \mathrm{M}$ and $0.75 \mu \mathrm{M}$ CoFeSP ${ }^{\ddagger}$ were rapidly mixed with increasing RACo concentrations $(2.5-30 \mu \mathrm{M})$, satisfying pseudo-first order conditions. Acceptor fluorescence increased rapidly upon RACo addition (Figure 3A). 
A

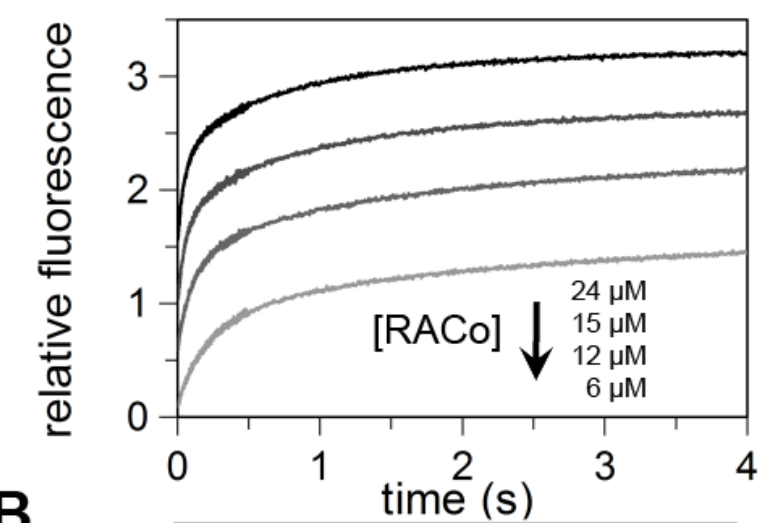

B

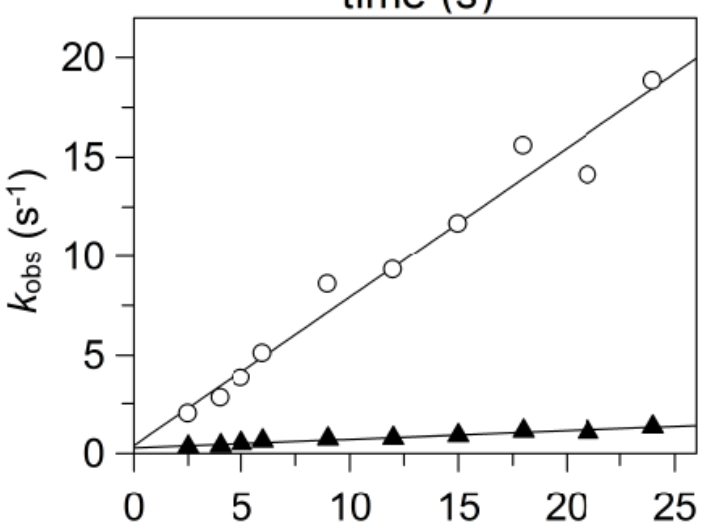

C

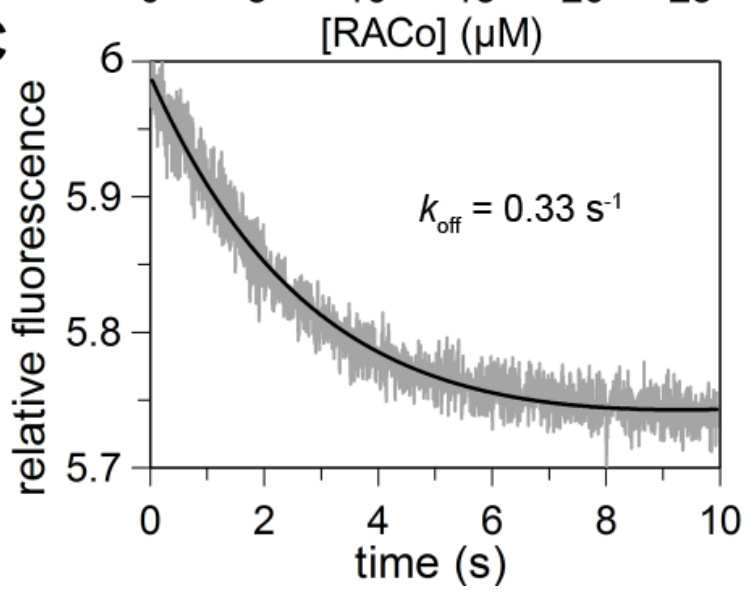

Figure 3. Transient kinetics of formation and dissociation of the RACo:CoFeSP complex. (A) Averaged transients of Atto 590 fluorescence for the reaction of $6,12,15$ and $24 \mu \mathrm{M}$ RACo with CoFeSP ${ }^{\ddagger}(0.25$ or $0.75 \mu \mathrm{M})$. (B) Dependency of the observed rate constants on RACo concentration. Observed rate constants were fitted using equation 4 . Circles denote the larger rate constant giving $k_{\text {on-fast }}=0.75 \pm$ $0.05 \mu \mathrm{M}^{-1} \mathrm{~s}^{-1}$ and $k_{\text {off-fast }}=0.39 \pm 0.67 \mathrm{~s}^{-1}$, filled triangles denote the smaller rate constant and are fitted with $k_{\text {on-slow }}=0.043 \pm 0.003 \mu \mathrm{M}^{-1} \mathrm{~s}^{-1}$ and $k_{\text {off-slow }}=0.28 \pm 0.04 \mathrm{~s}^{-1}$. (C) RACo:CoFeSP ${ }^{\ddagger}$ complex dissociation by competitive displacement. $1 \mu \mathrm{M}$ of RACo:CoFeSP ${ }^{\ddagger}$ complex was rapidly mixed with $50 \mu \mathrm{M}$ unlabeled CoFeSP. The decay of Atto 590 fluorescence was monitored after excitation at $450 \mathrm{~nm}$. The averaged curve of three experiments is plotted together with a mono-exponential fit (solid line). All experiments were carried out at $298 \mathrm{~K}$. 
The transients were fit by a double exponential function (equation 2), resulting in two observed rate constants differing by one order of magnitude but similar amplitudes (Figure $\mathrm{S6C}$ ). Both rate constants depend linearly on the RACo concentration (Figures 3B and Figure S6B). A linear fit of the observed rate constants (equation 4) for the fast phase gives an association rate constant ( $k_{\text {on-fast}}$ ) of $0.75 \pm 0.05 \mu \mathrm{M}^{-1} \mathrm{~s}^{-1}$ and an apparent dissociation rate constant $\left(k_{\text {off-fast }}\right)$ of $0.39 \pm 0.67 \mathrm{~s}^{-1}$, while giving for the slow phase an association rate constant ( $k_{\text {on-slow }}$ ) of $0.043 \pm 0.003 \mu \mathrm{M}^{-1} \mathrm{~s}^{-1}$ and an apparent dissociation rate constant $\left(k_{\text {off- }}\right.$ slow) of $0.28 \pm 0.04 \mathrm{~s}^{-1}$ (Figure 3B). As the error of the apparent $k_{\text {off-fast }}$ is larger than the number, it remained ill-defined in the association assay and we performed a competitive displacement experiment to determine it (Figure $3 \mathrm{C}$ ). The RACo:CoFeSP ${ }^{\ddagger}$ complex was rapidly mixed with a 50-fold molar excess of unlabeled CoFeSP. As complex formation between RACo and unlabeled COFeSP is FRET silent, only the dissociation of bi-labeled RACo:CoFeSP ${ }^{\ddagger}$ complex contributes to the decay of Atto 590 fluorescence after excitation at $450 \mathrm{~nm}$. Kinetic traces from three experiments were recorded (Figure S7) and averaged. The decrease in fluorescence was approximated with a single exponential function allowing us to refine the value of the dissociation rate constant $\left(k_{\text {off }}\right)$ to $0.33 \pm 0.05 \mathrm{~s}^{-1}$, which is within the range determined for the two phases in the association assay $\left(k_{\text {off-fast }}=0.39 \mathrm{~s}^{-1} ; k_{\text {off-slow }}=0.28\right.$ $\left.\mathrm{s}^{-1}\right)$. As a single exponential function sufficed to fit the competitive displacement assay and the determined $k_{\text {off }}$ value is within the error margin of both apparent dissociation rate constants from the association assay, we assume it to be more reliable.

As $k_{\text {obs }}$ values increase linearly with the concentration of RACo, we address the calculated kinetic parameters to the formation of the RACo:CoFeSP encounter complex. Although the formation of the encounter complex should not result in a change of the FRET signal, once it is rate-limiting for the following FRET sensitive conformational changes, it produces the observed linear increase of $k_{\text {obs }}$ with RACo concentration. In contrast, a rapid association step followed by a slower reorientation step would have resulted in a hyperbolic dependence of the $k_{\text {obs }}$ values on the RACo concentration, which was not observed. The two dissociation equilibrium constants resulting from the association and dissociation rate constants $\left(K_{\text {d-fast }}=k_{\text {off }} / k_{\text {on-fast }}=0.44 \mu \mathrm{M}\right.$ and $\left.K_{\text {d-slow }}=k_{\text {off }} / k_{\text {on-slow }}=7.67 \mu \mathrm{M}\right)$ are $23-$ and $400-$ times larger than the dissociation constant determined earlier by ITC. ${ }^{8}$

\section{DISCUSSION}


Recent crystal structures of the RACo:CoFeSP complex and the structure of RACo and CoFeSP alone indicated that both proteins undergo large conformational movements to form the complex (Figure 1). The changes are especially pronounced for the cobalamin-containing domain of CoFeSP, which relocates such that in the complex the $\beta$-face of the corrin ring faces the surface of RACo and $\mathrm{Co}^{2+}$, before having a water as a $\beta$-ligand now binds to a serine residue (Ser398) of RACo. ${ }^{8}$ The change in the PELDOR signal likely originates from these conformational changes, because (I) the observed distance change for the labeled positions is the nearly identical in the crystal structure and in solution and (II) the crystal structures could be used to model the distance distribution of the spin label.

In addition to the static crystal structure and the equilibrium distance distribution obtained from the PELDOR experiment, we also obtained insights into the dynamic of the conformational changes using identical engineered cysteine residues in both experiments. The observed biphasic transients in the FRET experiment were interpreted to arise from an inhomogeneity. Although biphasic transients in binding kinetics may also indicate a more complex multi-step binding mechanism, ${ }^{14}$ as both observed rate constants increase linearly with RACo concentration, enzyme inhomogeneity appears the most likely reason. This inhomogeneity probably derives from the CoFeSP preparation, which is prone to contain different Co oxidation states. ${ }^{9}$ As the FRET labels and their disulfide linkage to CoFeSP* are sensitive to reducing agents, we had to exclude them after labelling, most likely resulting in a mixture of $\mathrm{Co}^{2+}$ and $\mathrm{Co}^{3+} .{ }^{9}$ Complex formation between RACo and CoFeSP is strongest for Co(II)-CoFeSP, whereas Co(III)-CoFeSP forms only weak complexes with RACo. ${ }^{2}$ This could implicate that the larger $k_{\text {on }}\left(k_{\text {on-fast }}\right.$; smaller $K_{\mathrm{d}}$ ) derives from Co(II)-CoFeSP and the smaller $k_{\text {on }}\left(k_{\text {on-slow; }}\right.$ higher $K_{\mathrm{d}}$ ) from Co(III)-CoFeSP. The bimodality of the distance distribution observed in the PELDOR experiment may be due to this inhomogeneity, but may as well be due to the flexibility of the MTSL linker, as the protein structure-based simulations of rotamer distributions indicate.

The dynamic data complement our structural data and help to place complex formation into the catalytic cycle. The observed conformational changes are large, but not rate-limiting. Rate constants of $0.75 \mu \mathrm{M}^{-1} \mathrm{~s}^{-1}\left(0.043 \mu \mathrm{M}^{-1} \mathrm{~s}^{-1}\right.$ for the slow phase) and $0.33 \mathrm{~s}^{-1}$ for $k_{\text {on }}$ and $k_{\text {off }}$ show that formation of the RACo:CoFeSP encounter complex as well as the movement of the B12-binding domain of CoFeSP are fast compared to the rate of ATP hydrolysis by RACo (turnover number of $2.59 \mathrm{~min}^{-1}-$ in the presence of CoFeSP) and electron transfer. ${ }^{8}$ Thus, 
under our assay conditions using low $\mu \mathrm{M}$ concentrations of RACo and CoFeSP, complex formation between both proteins and the following conformational changes are not limiting the rate of reductive activation. Therefore other factors, such as ATP-binding and -hydrolysis are most likely limiting the rate of the reductive activation cycle.

During its reactivation and methyltransfer cycle, CoFeSP forms individual complexes with RACo, methyltransferase and acetyl-CoA synthase. ${ }^{1}$ Each time it needs to position its B12binding domain in close contact to the partner proteins and conformational changes for this domain are indeed similar in complexes of CoFeSP with RACo ${ }^{8}$ and with methyltransferase. ${ }^{19}$, 20 Our study reveals that CoFeSP is well adapted to its role as a methyltransfer agent with multiple partners by supporting rapid conformational changes of its B12-binding domain.

\section{ACKNOWLEDGMENT}

Rainer Dietrich is acknowledged for expert technical assistance.

\section{SUPPORTING INFORMATION PARAGRAPH}

Supporting information contains further information on the calculation of the PELDOR distance distributions, complex formation between RACo and CoFeSP*, ATP-dependent electron transfer, error validation of the distance distributions, a UV/Vis absorption spectrum of CoFeSP and kinetic traces from the association and displacement experiments. Supporting Figures are available under XXX.

\section{REFERENCES}

[1] Ragsdale, S. W., and Pierce, E. (2008) Acetogenesis and the Wood-Ljungdahl pathway of CO2 fixation, Bba-Proteins Proteom 1784, 1873-1898.

[2] Hennig, S. E., Jeoung, J. H., Goetzl, S., and Dobbek, H. (2012) Redox-dependent complex formation by an ATP-dependent activator of the corrinoid/iron-sulfur protein, $P$ Natl Acad Sci USA 109, 5235-5240.

[3] Schilhabel, A., Studenik, S., Vodisch, M., Kreher, S., Schlott, B., Pierik, A. J., and Diekert, G. (2009) The Ether-Cleaving Methyltransferase System of the Strict Anaerobe Acetobacterium dehalogenans: Analysis and Expression of the Encoding Genes (vol 191, pg 588, 2009), J Bacteriol 191, 1994-1994.

[4] Ferguson, T., Soares, J. A., Lienard, T., Gottschalk, G., and Krzycki, J. A. (2009) RamA, a Protein Required for Reductive Activation of Corrinoid-dependent Methylamine Methyltransferase Reactions in Methanogenic Archaea, J Biol Chem 284, 22852295.

[5] Sperfeld, M., Diekert, G., and Studenik, S. (2014) Kinetic regulation of a corrinoidreducing metallo-ATPase by its substrates, Mol Microbiol 92, 598-608. 
[6] Nguyen, H. D., Studenik, S., and Diekert, G. (2013) Corrinoid activation by a RACE protein: studies on the interaction of the proteins involved, Fems Microbiol Lett 345, 31-38.

[7] Meister, W., Hennig, S. E., Jeoung, J. H., Lendzian, F., Dobbek, H., and Hildebrandt, P. (2012) Complex Formation with the Activator RACo Affects the Corrinoid Structure of CoFeSP, Biochemistry-Us 51, 7040-7042.

[8] Hennig, S. E., Goetzl, S., Jeoung, J. H., Bommer, M., Lendzian, F., Hildebrandt, P., and Dobbek, H. (2014) ATP-induced electron transfer by redox-selective partner recognition, Nat Commun 5.

[9] Schrapers, P., Mebs, S., Goetzl, S., Hennig, S. E., Dau, H., Dobbek, H., and Haumann, M. (2016) Axial Ligation and Redox Changes at the Cobalt Ion in Cobalamin Bound to Corrinoid Iron-Sulfur Protein (CoFeSP) or in Solution Characterized by XAS and DFT, Plos One 11.

[10] Goetzl, S., Jeoung, J. H., Hennig, S. E., and Dobbek, H. (2011) Structural Basis for Electron and Methyl-Group Transfer in a Methyltransferase System Operating in the Reductive Acetyl-CoA Pathway, J Mol Biol 411, 96-109.

[11] Svetlitchnaia, T., Svetlitchnyi, V., Meyer, O., and Dobbek, H. (2006) Structural insights into methyltransfer reactions of a corrinoid iron-sulfur protein involved in acetyl-CoA synthesis, $P$ Natl Acad Sci USA 103, 14331-14336.

[12] Emsley, P., and Cowtan, K. (2004) Coot: model-building tools for molecular graphics, Acta Crystallogr D 60, 2126-2132.

[13] Jeoung, J. H., and Dobbek, H. (2007) Carbon dioxide activation at the Ni,Fe-cluster of anaerobic carbon monoxide dehydrogenase, Science 318, 1461-1464.

[14] Johnson, K. A. (1992) Transient-State Kinetic Analysis of Enzyme Reaction Pathways, In The enzymes (Sigman, D. S., Ed.), pp 1 - 61, Academic Press.

[15] Thomä, N., and Goody, R. S. (2003) What to do if there is no signal: using competition experiments to determine binding parameters, In Kinetic Analysis of Macromolecules (Johnson, K. A., Ed.), pp 153-178, Oxford University Press, Oxford.

[16] Pannier, M., Veit, S., Godt, A., Jeschke, G., and Spiess, H. W. (2000) Dead-time free measurement of dipole-dipole interactions between electron spins, J Magn Reson 142, 331-340.

[17] Jeschke, G., Chechik, V., Ionita, P., Godt, A., Zimmermann, H., Banham, J., Timmel, C. R., Hilger, D., and Jung, H. (2006) DeerAnalysis2006 - a comprehensive software package for analyzing pulsed ELDOR data, Appl Magn Reson 30, 473-498.

[18] Polyhach, Y., Bordignon, E., and Jeschke, G. (2011) Rotamer libraries of spin labelled cysteines for protein studies, Phys Chem Chem Phys 13, 2356-2366.

[19] Kung, Y., Ando, N., Doukov, T. I., Blasiak, L. C., Bender, G., Seravalli, J., Ragsdale, S. W., and Drennan, C. L. (2012) Visualizing molecular juggling within a B12-dependent methyltransferase complex, Nature 484, 265-269.

[20] Ando, N., Kung, Y., Can, M., Bender, G., Ragsdale, S. W., and Drennan, C. L. (2012) Transient B12-dependent methyltransferase complexes revealed by small-angle X-ray scattering, J Am Chem Soc 134, 17945-17954. 
Graphic for the Table of Contents

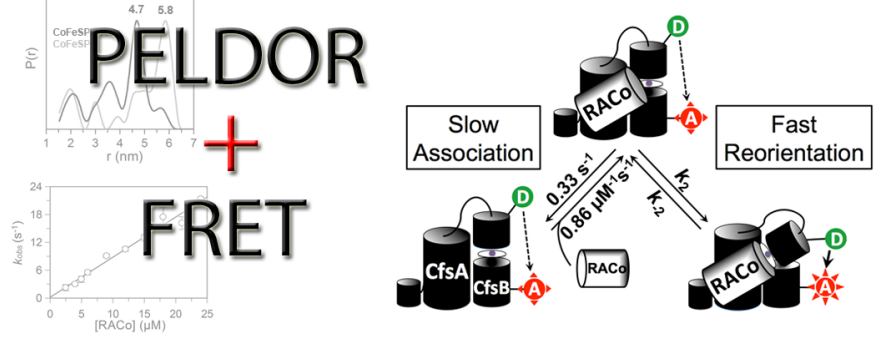

